\title{
VDDDAs-based Voltage-mode Multiphase Sinusoidal Oscillator
}

\author{
Sunti Tuntrakool ${ }^{\mathrm{a},{ }^{*}}$, Montree Kumngern ${ }^{\mathrm{a}}$, Winai Jaikla ${ }^{\mathrm{b}}$ \\ ${ }^{\mathrm{a}}$ Department of Telecommunications Engineering, Faculty of Engineering, \\ King Mongkut's Institute of Technology Ladkrabang, Bangkok 10520, Thailand \\ ${ }^{b}$ Department of Engineering Education, Faculty of Industrial Education, \\ King Mongkut's Institute of Technology Ladkrabang, Bangkok 10520, Thailand \\ *Corresponding Author: sunti.tu@kmitl.ac.th
}

\begin{abstract}
The design of voltage-mode multiphase sinusoidal oscillator (MSO) is presented. The odd phase system can be realized using voltage differencing differential difference amplifier (VDDDA)-based lossy integrators. The condition of oscillation and frequency of oscillation can be controlled independently through adjusting the bias current of the VDDDA. The low output impedances facilitate easy driving an external load without additional voltage buffers. The proposed MSO provides odd phase signals that are equally spaced in phase and equal amplitude. The circuit requires one VDDDA, two resistors and one grounded capacitor per phase without external resistor and additional current amplifier. The results of PSPICE simulations using $0.18 \mu \mathrm{s}$ TSMC CMOS technology are included to verify theory.
\end{abstract}

Keywords: VDDDA, multiphase sinusoidal oscillator, Integrated Circuit.

\section{Introduction}

Multiphase sinusoidal oscillator (MSO) is important blocks for various applications. For example, in telecommunications it is used for phase modulators, quadrature mixers $^{(1)}$, and single-sideband generators ${ }^{(2)}$. In measurement system, MSO is employed for vector generator or selective voltmeters ${ }^{(3)}$. It can also be utilized in power electronics systems ${ }^{(4)}$. Recently, current-mode circuits have been receiving considerable attention of due to their potential advantages such as inherently wide bandwidth, lower slew-rate, greater linearity, wider dynamic range, simple circuitry and low power consumption $^{(5)}$. Many active building blocks (ABBs) have been proposed to realize the current-mode circuit. The interesting active element, called current controlled current differencing transconductance amplifier $(\text { CCCDTA })^{(6-7)}$, is introduced to provide new possibilities in the current-mode circuit. It is really current-mode element whose input and output signal are currents. In addition, output currents of CCCDTA can be electronically adjusted.

Several realizations of current-mode MSOs using different active building blocks are available in the literature. These include realizations using current follower $(\mathrm{CF})^{(8)}, \mathrm{CCCII}^{(9),(11)}, \mathrm{CDTA}^{(12),(14)}, \mathrm{CDBA}^{(15)}, \mathrm{CFOA}^{(16)}$, and $\mathrm{CCCCTA}^{(17)}$ and $\mathrm{CCCDTA}^{(18-19)}$. The CF-based $\mathrm{MSO}^{(8)}$ requires two current followers, one floating resistor, and one floating capacitor for each phase and thus the circuit is not suitable for monolithic integration. Moreover, it cannot be electronically controlled. The CCCII-based MSOs ${ }^{(9),(11)}$ enjoy high-output impedances and electronic tunability. However, the first one requires a large number of external capacitors. In addition, the oscillation condition can be provided by tuning the capacitance ratio of external capacitors, which is not easy to implement. The second reported circuit requires additional current amplifiers, which makes the circuit more complicated and increases its power consumption. CDTA-based current-mode $\mathrm{MSOs}^{(12)}$ is based on lossy integrators, whereas the circuits ${ }^{(13-14)}$ contain CDTA-based allpass sections. They exhibit good performance in terms of electronic tunability, high-output impedances, and independent control of the oscillation frequency and the oscillation condition. However, MSOs $^{(12-13)}$ require an additional current amplifier, which is implemented by two CDTAs. Moreover, the output currents of the MSO, utilizing the CDTA-based lossy integrators, are of different amplitudes. The MSO employing CDTA-based 
allpass sections ${ }^{(13)}$ requires two CDTAs in each allpass section, and the circuitry becomes more extensive. While MSO using CDTA-based allpass sections $^{(14)}$ requires floating capacitor. Consequently, it occupies a larger chip area for VLSI design. In addition, its power consumption is also increased.

The purpose of this study is to introduce a new current-mode multiphase sinusoidal oscillator. The features of the proposed circuit are the following: (I) Use of grounded capacitors and identical circuit configuration for each section in the MSO topology which are suitable for integration. (II) The electronic tunability of oscillation condition and oscillation frequency. (II) High-impedance current outputs. (IV) The possibility of generating multi-phase signals for both an even and odd number of equally-spaced in phases. (V) Independent tuning of the oscillation frequency and the oscillation condition. (VI) Equality of amplitudes of each phase due to utilizing identical sections. (VII) Requirement for only one CCCDTA as the active element for each phase without any additional current amplifiers.

\section{Proposed Multiphase Sinusoidal Oscillator}

First, the characteristic of the main active building block will be described. It is named as voltage differencing differential difference amplifier (VDDDA). This device is formed by operational transconductance amplifier (OTA) followed by voltage differencing differential difference unit (VDDDU). The symbol and equivalent circuit of VDDDA are given in figure 1(a) and 1(b), respectively. It has high impedance input voltage terminals $\left(\mathrm{V}_{+}, \mathrm{V}_{-}, \mathrm{V}_{\mathrm{p}}\right.$, and $\left.\mathrm{V}_{\mathrm{n}}\right)$, high impedance output current terminal (z) and low impedance output voltage terminal (w). The difference of input voltage $\mathrm{V}+$ and $\mathrm{V}$ - will be send to be current $\mathrm{I}_{\mathrm{z}}$ via transconductance $\left(g_{m}\right)$. Generally, the $g_{m}$ is varied and electronically tuned and the differential voltage of $\mathrm{V}_{\mathrm{z}}, \mathrm{V}_{\mathrm{n}}$ and $V_{p}$ will be send to be voltage $V_{w}$ with the unity voltage gain. The idea VDDDA is characterized by following equations:

$$
\left(\begin{array}{l}
I_{v+} \\
I_{v-} \\
I_{z} \\
I_{v p 1} \\
I_{v p 2} \\
V_{w}
\end{array}\right)=\left(\begin{array}{cccccc}
0 & 0 & 0 & 0 & 0 & 0 \\
0 & 0 & 0 & 0 & 0 & 0 \\
g_{m} & -g_{m} & 0 & 0 & 0 & 0 \\
0 & 0 & 0 & 0 & 0 & 0 \\
0 & 0 & 0 & 0 & 0 & 0 \\
0 & 0 & -1 & 1 & 1 & 0
\end{array}\right)\left(\begin{array}{l}
V_{v+} \\
V_{v-} \\
V_{z} \\
V_{v p 1} \\
V_{v p 2} \\
I_{w}
\end{array}\right)
$$

For the VDDDA implemented by CMOS technology, the $g_{m}$ is depends on both physical parameters of CMOS transistor and bias current $\left(\mathrm{I}_{\mathrm{B}}\right)$ and is given as

$$
g_{m}=\sqrt{\mu C_{o x}\left(\frac{W}{L}\right)_{1,2} I_{B}}
$$

where $\mu$ is the mobility of the carrier for NMOS transistors $\left(M_{1}\right.$ and $M_{2}$ in figure $\left.1(c)\right), C_{o x}$ is the gate oxide capacitance per unit area, $\mathrm{W}$ and $\mathrm{L}$ are the channel width and channel length, respectively. The internal construction of CMOS VDDDA is shown in figure 1(c).

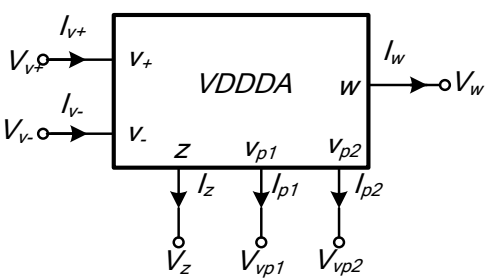

(a)

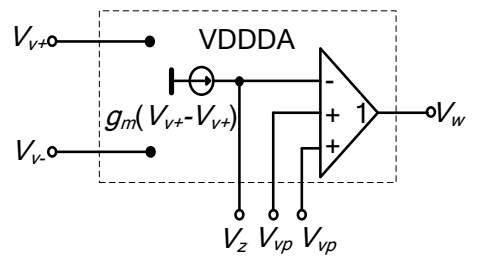

(b)

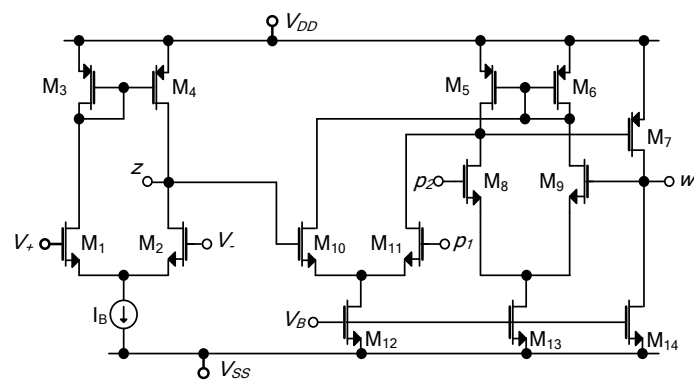

(c)

Fig. 1 (a) Symbol (b) equivalent circuit of VDDDA (c) Internal construction of VDDDA

The generalized structure of MSO is designed by cascading the $n$ identical stages ( $\mathrm{n} \geq 3$ and $n$ is odd number) which contains the lossy integrator (first order low pass filter) for each phase as shown in figure 2, where $a$ is the time constant and $k$ is the gain. The output of $n^{\text {th }}$ stage is fed back to the input of the first stage. The system can provide 
one phase per one lossy integrator without any additional external amplifier. The oscillator based on this block diagram is available for only odd phase. If $n$ is the number of phase, then $\mathrm{n}$ has to be $3,5,7, \ldots$ Based on block diagram in figure 2 , the frequency of oscillation (FO) and condition of oscillation $(\mathrm{CO})$ are written as

$$
F O: \omega_{o s c}=\frac{1}{a} \tan \frac{\pi}{n}
$$

and

$$
C O: k \geq \sec \frac{\pi}{n}
$$

It is found from Equations (equations 3 and 4) that the frequency of oscillation and condition of oscillation are independent controlled.

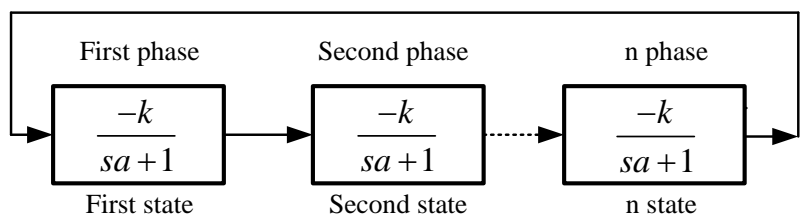

Fig. 2 Lossy integrator based MSO

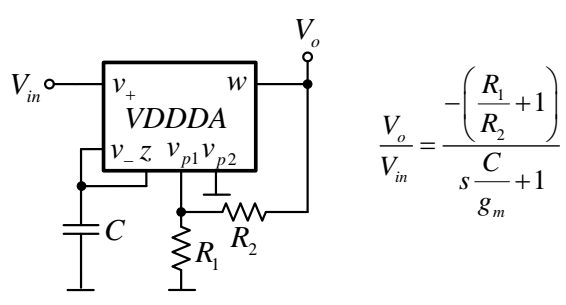

(a)

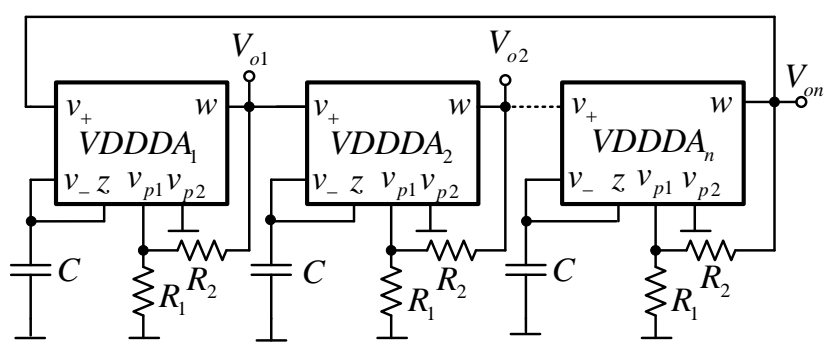

(b)

Fig. 3 (a) The VDDDA-based lossy integrator

(b) Proposed MSO

The VDDDA-based lossy integrator is shown in figure 3(a). The proposed voltage-mode odd phase MSO is shown in figure 3(b). It is found from figure 3(b) that the current mirrors are required to split the bias currents $I_{B}$ to each lossy integrator section. In addition, it can be seen that the proposed MSO enjoy low output impedances which facilitate easy driving an external load without additional voltage buffers. From circuit in figure 3(b) for $n=3,5,7, \ldots$, the frequency of oscillation (FO) and condition of oscillation $(\mathrm{CO})$ are expressed as equations 5 and 6 respectively $^{(16)}$.

$$
\begin{aligned}
& F O: \omega_{o s c}=\frac{g_{m}}{C} \tan \frac{\pi}{n} \\
& C O: \frac{R_{1}}{R_{2}}+1 \geq \sec \frac{\pi}{n}
\end{aligned}
$$

From equation 5 , if $\mathrm{g}_{\mathrm{m}}$ is equal to equation 2 , the $\mathrm{FO}$ is modified as

$$
\omega_{o s c}=\sqrt{\mu C_{o x}\left(\frac{W}{L}\right)_{1,2} I_{B}} \tan \frac{\pi}{n}
$$

From equation 7 , it can be seen that the FO can be adjusted electronically/independently from the $\mathrm{CO}$ by varying $I_{B}$.

\section{Simulation Results}

PSPICE simulation using CMOS schematic of VDDDA as given in figure 1(c) which was modified from (7) was performed in order to verify the theoretical analysis of the proposed oscillator. In this simulation of PMOS and NMOS transistors, Parameters of a $0.18 \mu \mathrm{m}$ TSMC CMOS technology (level 7) [20] with $\pm 0.9 \mathrm{~V}$ voltage supply and $\mathrm{V}_{\mathrm{B}}=-0.35 \mathrm{~V}$ was used. Aspect ratios of PMOS and NMOS transistors are listed in (20) as follow. Parameters used for designing of the proposed oscillator were as follows: $\mathrm{C}_{1}=$ $33 \mathrm{pF}, \mathrm{I}_{\mathrm{B}}=50 \mu \mathrm{A}, \mathrm{R}_{1}=10 \mathrm{k} \Omega$ and $\mathrm{R}_{2}=9.9 \mathrm{k} \Omega$. An odd three-phase sinusoidal oscillator $(n=3)$ based on the structure in figure 3(b) has been designed. The simulated output waveforms, $\mathrm{V}_{\mathrm{O} 1}, \mathrm{~V}_{\mathrm{O} 2}$ and $\mathrm{V}_{\mathrm{O} 3}$ are shown in figure 4 . The frequency of oscillation achieved from the simulation was $2.5 \mathrm{MHz}$. The frequency spectrum of output voltages are shown in figure 5. The total harmonic distortion for $\mathrm{V}_{\mathrm{O} 1}$, $\mathrm{V}_{\mathrm{O} 2}$ and $\mathrm{V}_{\mathrm{O} 3}$ are $0.87 \%, 0.86 \%$ and $0.78 \%$ respectively. 


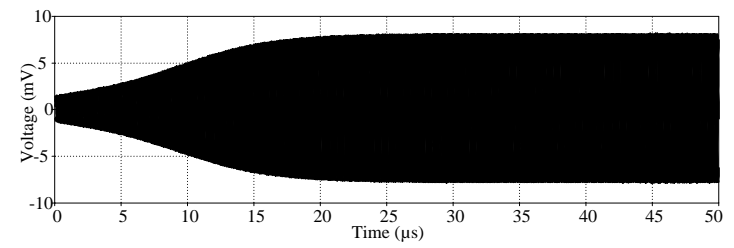

(a)

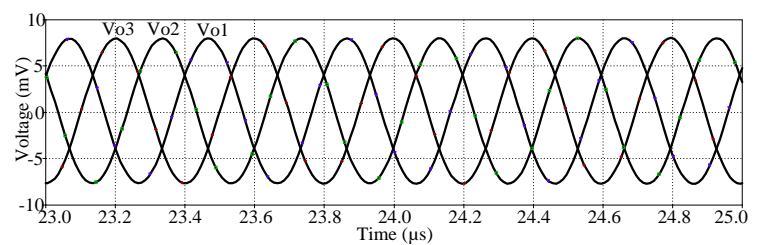

(b)

Fig. 4 Sinusoidal output voltages (a) Initial state

(b) Steady state

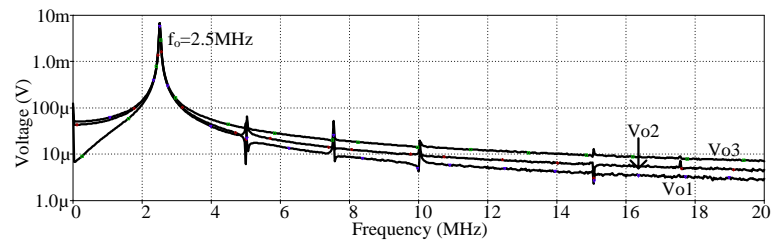

Fig. 5 Output Spectrum

\section{Conclusions}

New voltage-mode multiphase sinusoidal oscillators using VDDDA-based lossy integrators with grounded capacitors have been presented. The features of the proposed circuit are that: oscillation frequency and oscillation condition can be independently tuned; the proposed oscillator consists of merely 1 VDDDA, 2 resistor and 1 grounded capacitor for each phase and no additional current amplifier and availability of explicit-current outputs from low-output impedance terminals. PSPICE simulation results agree well with the theoretical anticipation.

\section{References}

(1) J. Dunlop and D.G.Smith, "Telecommunications Engineering," 3rd ed. CRC Press, 1994

(2) W. Tomasi, "Electronic Communications System," New Jersey: Prentice- Hall Inc., 1998.

(3) Anritsu Emea Limited, "MG3700A vector signal generator datasheet," Availableat: http://www.eu.anritsu.com/files/MEG3700A El17301.pdf.
(4) G. Eirea, and S.R. Sanders, "Phase current unbalance estimation in multiphase buck converters," IEEE Transactions on Power Electronics, vol. 23, pp. 137-143, 2008.

(5) C. Toumazou, F.J. Lidgey and D.G. Haigh, "Analogue IC design: the current-mode approach," Peter Peregrinus: London, (1990).

(6) D. Biolek, "CDTA - Building block for current-mode analog signal processing," Proceedings of the European Conference on Circuit Theory and Design 2003 - ECCTD'03, Krakow, Poland, pp. 397-400, 2003.

(7) N. Herensar; R. Sotner, B. Metin, J. Koton and K. Vrba, "VDDDA - New 'voltage differencing' device for analog signal processing," Proceeding of the 8th International Conference on Electrical and Electronics Engineering. Bursa, Turkey: The Chamber of Electrical Engineers Bursa Branch (EMO), 2013. pp. 17-20.

(8) M.T. Abuelma'Atti, "Current-mode multiphase oscillator using current followers" Microelectronics Journal, vol. 25, 457-461, 1994.

(9) G D.Skotis, and C. Psychalinos, "Multiphase sinusodal oscillators using second generation current conveyors," Int. J. Electron. Commu. (AEU), vol.64, pp.1178-1181, 2010.

(10) M.T. Abuelma'atti, and M.A.Al-Qahtani, "A new current- controlled multiphase sinusoidal oscillator using translinear current conveyor," IEEE Transactions on Circuits and Systems, vol. 45, 881$885,1998$.

(11)C. Loescharataramdee, W.Kiranon, W.Sangpisit and W.Yadum, "Multiphase sinusoidal oscillators using translinear current conveyors and only grounded passive components," Proceeding of Southeastern Symposium on System Theory, 2004, 59-63.

(12) W. Tangsrirat and W. Tanjaroen, "Current-mode multiphase sinusoidal oscillator using current differencing transconductance amplifiers," Circuits, Systems and Signal Processing, vol. 27, pp. 81- 93, 2008.

(13) W. Tangsrirat, W. Tanjaroen, and T. Pukkalanun, "Current-mode multiphase sinusoidal oscillator using CDTA-based allpass sections," International Journal of Electronics and Communications (AEU), vol. 63, pp. 616- 622, 2009. 
(14)W. Jaikla, M. Siripruchyanun, D. Biolek, and V. Biolkova, "High-output-impedance current-mode multiphase sinusoidal oscillator employing current differencing transconductance amplifier-based allpass filters, " International Journal of Electronics, vol. 97, pp. 811-826, 2010.

(15) K. Klahan, W. Tangsrirat, and W. Surakampontorn, "Realization of multiphase sinusodal oscillator using CDBAs," IEEE Asia-Pacific Conf. Circ. Sys., pp. 725-728, 2004.

(16)D.-S. Wu, S.-I. Liu, Y.-S. Hwang and Y.-P. Wu, "Multiphase sinusoidal oscillator using the CFOA pole," IEE Proc. Circuits Devices Syst, vol.142, pp. 37-40, 1995.

(17) P. Uttaphut, "New Current-mode Multiphase Sinusoidal Oscillators Based on CCCCTA-based Lossy Integrators," Electrical Review, pp. 291-295, 2012.

(18) M. Kumngern, "Current-mode multiphase sinusoidal oscillator using current-controlled current differencing transconductance amplifiers," 2010 IEEE International Conference of Electron Devices and Solid-State Circuits (EDSSC), 2010.

(19) W. Jaikla, and P. Prommee, "Electronically tunable current-mode multiphase sinusoidal oscillator employing CCCDTA-base allpass filters with only grounded passive elements," Radioengineering, vol.20, no.3, pp. 594-599, 2011.

(20) http://lgjohn.ecen.ceat.okstate.edu/5263/processparam/t 4bk lo epi-params.html 